\title{
Robust Mean Absolute Deviation Problems on Networks with Linear Vertex Weights
}

\author{
M. C. López-de-los-Mozos \\ Departamento de Matemática Aplicada I. Universidad de Sevilla, Spain
}

\author{
J. Puerto \\ Departamento de Estadística e Investigación Operativa. Universidad de Sevilla, Spain
}

\author{
A. M. Rodríguez-Chía \\ Departamento de Estadística e Investigación Operativa. Universidad de Cádiz, Spain
}

\begin{abstract}
This article deals with incorporating the mean absolute deviation objective function in several robust single facility location models on networks with dynamic evolution of node weights, which are modeled by means of linear functions of a parameter. Specifically, we have considered two robustness criteria applied to the mean absolute deviation problem: the MinMax criterion, and the MinMax regret criterion. For solving the corresponding optimization problems, exact algorithms have been proposed and their complexities have been also analyzed.
\end{abstract}

Keywords: location; networks; robust optimization; equality measures

\section{INTRODUCTION}

Standard network location models deal with problems of locating facilities on a network optimizing some objective function that involves distances from nodes to the facilities. Edge lengths and node weights are parameters in the model and they are very often uncertain. Because there are a wide range of causes for the uncertainty there exist several approaches to deal with it in optimization models, see

Contract grant sponsor: Spanish Ministry of Science and Innovation/ FEDER; Contract grant numbers: MTM2007-67433-C02-(01,02), MTM2009-14243, MTM2010-19576-C02-(01,02), DE2009-0057 Contract grant sponsor: Fundación Séneca; Contract grant number: 08716/ $\mathrm{PI} / 08$

Contract grant sponsor: Junta de Andalucía/FEDER; Contract grant numbers: P09-TEP-5022, FQM-5849 for instance Kouvelis and $\mathrm{Yu}$ [20]. A possible approach to tackle uncertainty within this framework is by adopting a minimax regret point of view. The regret or savage function represents the opportunity loss and is the difference between the objective function value and the optimal objective function value under the realized scenario. Although the resulting criterion is a conservative one, it is not as pessimistic as pure minmax. The state-of-the-art of the minmax regret discrete optimization as well as a discussion on its potential applications up to 1997 is contained in the book by Kouvelis and $\mathrm{Yu}$ [20]. Minmax regret optimization has been widely applied to different location models as: median, center, and cent-dian objective functions on networks (Averbakh [1-3], Averbakh and Berman [4-7], Averbakh and Lebedev [8], Burkard and Dollani [10,11], Chen and Lin [14], Conde [16, 17], Ogryczak [25], Puerto et al. [27, 28]).

In contrast, location problems in the public sector give rise to consider equity aspects of location (such as the dispersion of the distribution of distances from the facility to the demand points) which are not captured by either the median and center objective functions nor even a convex combination of them. Assuming that customers have the same utility function, the equity concept in location is usually quantified by means of measures which gauge the level of inequality of the distribution of distances, and the resulting problems attempt to minimize such an inequality. In this way, equity in location commonly represents "equality" (in fact both terms are often synonymously used in the literature). From among the 20 different measures described in Marsh and Schilling [22], the mean absolute deviation (MAD) is a measure of the absolute deviations of the customers from the average distance, and it has been widely studied by several authors with purposes of equity (see Berman and Kaplan [9], Tamir [30], Mesa et al. [23]). Models incorporating this inequality measure are structurally different from models based on the 
median function because, although both measures share the same "weighted sum" operator, the distance function vector considered in each model gives rise to different problems with different behavior and properties.

This article deals with the incorporation of the above inequality measure in a robust single-facility location model on a network when the data change dynamically. Specifically, we assume that the vertex weights are linear functions of a parameter, time for example, and we study several robust versions of the 1-mean absolute deviation (1-MAD) problem with respect to dynamic changes of the data. This type of dynamic model is studied by Vairaktarakis and Kouvelis [31] and Burkard and Dollani [10], which consider different robust versions of the 1-median problem on a tree that combine dynamically evolving and uncertain parameters. In this article, two of such robustness criteria have been applied to the mean absolute deviation objective: the MinMax criterion, whose aim is to minimize the largest objective value, and the MinMax regret (or the robust deviation) criterion, which minimizes the largest feasible deviation from the best objective value. The study of the corresponding optimization problems is the purpose of this work.

The remainder of the article is organized as follows: Section 2 introduces the notation and formulates these two problems. Section 3 is devoted to study the first one: the MinMax 1-MAD problem, in both a tree network and a cyclic network. For each case, an exact algorithm is proposed and its complexity discussed. Likewise, the MinMax regret 1MAD problem is studied and solved in Section 4 by also considering both a tree network and a cyclic network. Our conclusions together with some extensions of the models are summarized in Section 5. Finally, in the appendix of the article, we recall some known results used for our computational complexity analysis.

\section{PROBLEM FORMULATIONS}

Let $N(V, E)$ be a network with vertex set $V,|V|=n$ and edge set $E$, with $|E|=m$. Each vertex has associated a nonnegative weight representing the intensity of its demand. Each edge $e=[u, v]$ is represented by a continuous rectifiable arc with positive length $\ell_{e}$. Therefore any real number $x \in$ $\left[0, \ell_{e}\right]$ denotes the location in the edge $e=[u, v]$ for which the length of the subedge $[u, x]$ is $x$. We let $N$ denote the continuum set of points of the network. The edge lengths induce a distance function on $N$, such that the distance $d(x, y)$ between any two points $x, y$ in $N$ is defined as the length of a shortest path connecting $x$ and $y$. Therefore, $N$ is a metric space with respect the above distance function.

In this article, we consider that the weights are of dynamic nature and they are modeled by means of linear functions of a parameter $t$ varying in a given interval. This way, we consider each weight $w_{i}(t)=\alpha_{i} t+\beta_{i} \geq 0, i=1, \ldots, n$, where $t \in\left[t^{-}, t^{+}\right]$(lower and upper bounds for $t$, respectively). Each weight can be viewed as the demand rate at time $t$ (or other magnitude depending on a parameter $t$ ) at vertex $v_{i}, i=1, \ldots, n$. Let $W(t)=\sum_{i=1}^{n} w_{i}(t)=A t+B$ be the total weight, with $A=\sum_{i=1}^{n} \alpha_{i}$ and $B=\sum_{i=1}^{n} \beta_{i}$. Without loss of generality, we can assume that $W(t)>0$, for $t \in$ $\left[t^{-}, t^{+}\right]$. Henceforth, let us also consider $\left\{\frac{w_{i}(t)}{W(t)}, i=1, \ldots, n\right\}$ the normalized system of weights.

For $t$ in $\left[t^{-}, t^{+}\right]$, the dynamic median function is given by $M(x, t)=\frac{1}{W(t)} \sum_{i=1}^{n} w_{i}(t) d\left(v_{i}, x\right)$, and the dynamic MADfunction, by $F(x, t)=\frac{1}{W(t)} \sum_{i=1}^{n} w_{i}(t)\left|d\left(v_{i}, x\right)-M(x, t)\right|$. Therefore, for each $t$ the corresponding single facility location problems are formulated as $\operatorname{MED}(t): \min _{x \in N} M(x, t)$ and $\operatorname{MAD}(t): \min _{x \in N} F(x, t)$, respectively.

For each $t$, let $\phi F^{*}(t)=\min _{x \in N} F(x, t)$ be the optimal value of the $\operatorname{MAD}(t)$ problem. This solution is found on a cyclic network in $O(m n \log n)$ time by Tamir [30], and on a tree network in $O\left(n^{2}\right)$ time by Mesa et al. [23].

With these definitions, the incorporation of the dynamic MAD-function into the aforementioned robust models gives the following two problems:

1. The MinMax MAD location problem:

$$
\text { (MM-MAD) } \min _{x \in N} \max _{t \in\left[t^{-}, t^{+}\right]} F(x, t)
$$

2. The MinMax-Regret MAD location problem:

$$
\text { (MMR-MAD) } \min _{x \in N} \max _{t \in\left[t^{-}, t^{+}\right]}\left\{F(x, t)-F^{*}(t)\right\}
$$

Remark 1. Observe that in the formulations above, we have considered that the weights are linear functions of a parameter t to model the dynamic evolution in the data. However, we are looking for a unique solution in the entire interval $\left[t^{-}, t^{+}\right]$, an alternative interpretation of these dynamic weights is that demand of the nodes is linearly correlated and uncertain so that using the minimax criterion one looks for a robust solution simultaneously for all values of $t$.

We now analyze separately each of these problems.

\section{THE MINMAX MEAN ABSOLUTE DEVIATION PROBLEM}

In this section, we consider the (MM-MAD) location problem. We first study the problem on a tree network, and later the results will be applied to solve the problem on a general network.

\subsection{The Problem on a Tree Network}

Before solving the (MM-MAD) problem on a tree network, we are interested to know if the solutions of this problem (globally viewed) keep the same properties as the solutions obtained for this model with other objective functions.

For the median function, Vairaktarakis and Kouvelis [31] studied several dynamic robust problems on a tree with linear and positive weights, and they solved them using a result by Erkut and Tansel [19]. This result states that, on a tree 
network, the solutions of $\operatorname{MED}(t)$ problem belong to the unique path connecting the 1-median solutions of $\operatorname{MAD}\left(t^{-}\right)$ and $\operatorname{MAD}\left(t^{+}\right)$, respectively (nestedness property). Besides, such an interval can be reduced to a finite set of $t$-values for which all median solutions are obtained. However, this property does not hold for the $\operatorname{MAD}(t)$ problem, as the following two examples show. The first one is very simple and can be easily checked by the reader. The second one, borrowed from a talk based on an earlier version of this article presented at EWGLA XVIII (see [13]), is more sophisticate and it will be used to illustrate the results of the next sections.

Example 2. Let $v_{1}, v_{2}, v_{3}$ be tree vertices on the segment $[0,6]$ placed at the points 0,2 and 6 , respectively, and let $w_{1}(t)=t+2, w_{2}(t)=2 t-1, w_{3}(t)=-t+5$ be the respective linear weights, with $t \in[1,4]$. It is easy to see that the solutions of the problems $M A D(1)$ and $M A D(4)$ are located at the points $x^{*}(1)=13 / 4$ and $x^{*}(4)=5 / 4$ respectively, whereas the solution of the $M A D(3)$ problem is attained at the point $x^{*}(3)=7 / 2$, which is outside the segment $[5 / 4,13 / 4]$.

Example 3. Consider the tree network with eight vertices displayed in Figure 1, whose edge lengths are the numbers on the edges and whose linear weights $\left\{w_{i}(t), i=1, \ldots, 8\right\}$ are: $\{2 t+1,3 t-2,-t+8,4 t+3,-2 t+11,5 t+1,-3 t+$ $20,6 t-4\}$, with $t \in[1,4]$. (This example appears in [13].) The solutions of the problem $\operatorname{MAD}(t)$ for $t^{-}=1$ and $t^{+}=4$ are respectively the points $x^{*}\left(t^{-}\right)=7.3125$ and $x^{*}\left(t^{+}\right)=8.0$, both solutions lying in the edge $\left[v_{2}, v_{3}\right]$ and measuring the distance from the left vertex $v_{2}$. However, for $t=1.5$, the corresponding $\mathrm{MAD}(1.5)$ problem reaches the minimum at point $x^{*}(1.5)=11.9714$ of the edge $\left[v_{2}, v_{3}\right]$ (this point also measured from the left vertex $\left.v_{2}\right)$. Clearly, this last minimum is outside the path linking the 1-MAD solutions $x^{*}(1), x^{*}(4)$.

In addition, the dynamic median function for a fixed $\bar{x}, M(\bar{x}, t)$, is either strictly increasing, strictly decreasing or constant on the entire interval $\left[t^{-}, t^{+}\right]$. Consequently, the problem $\max _{t \in\left[t^{-}, t^{+}\right]} M(\bar{x}, t)$ attains its maximum value at either $t^{-}$or $t^{+}$. However, this does not occur with the MAD-function.

Indeed, for a fixed $\bar{x}$ the function $F(\bar{x}, t)=$ $\frac{1}{W(t)} \sum_{i=1}^{n} w_{i}(t)\left|d\left(v_{i}, \bar{x}\right)-M(\bar{x}, t)\right|$ is a continuous function of $t$, but in general it is neither concave nor convex when $t$ varies in the overall interval $\left[t^{-}, t^{+}\right]$. Besides, $F(\bar{x}, t)$ can have at most $n$ breakpoints on the $t$ argument, and each of the $n$ "pieces" composing $F(\bar{x}, t)$ can be nonlinear.

If we now consider a fixed value $\bar{t}$, the resulting function $F(x, \bar{t})$ is the classical MAD measure whose behavior and properties have already been widely studied (Berman, Kaplan [9], Mesa et al. [23], Tamir [30]). On each edge $e$ of the tree $F(x, \bar{t})$ is a continuous, convex and piecewise linear function with at most $n$ breakpoints. Each of such breakpoints is the intersection point of the median function $M(x, \bar{t})$ with some distance function. These breakpoints can be computed and sorted in $O(n \log n)$ time by using the procedure in Tamir [30]. Besides, the local solutions of the problem are reached

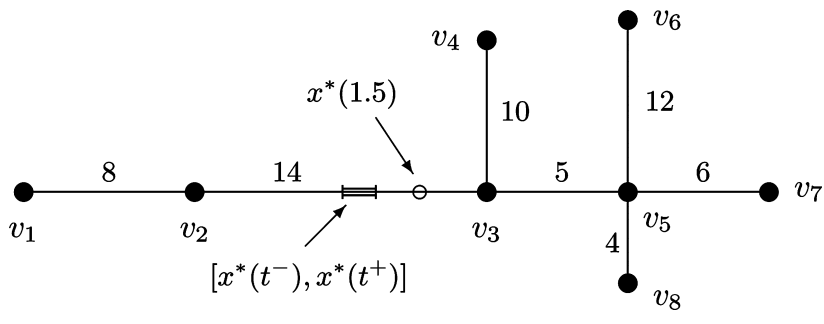

FIG. 1. Path linking the MAD-solutions for the extreme $t$-values.

at either one of the breakpoints or at the endpoints of the edge, which implies that the set of breakpoints (together with the endpoints of the edge) is a finite dominating set (FDS) for the restricted problem $\min _{x \in e} F(x, \bar{t})$. Note that from the structure of $F(x, \bar{t})$, the points of the FDS are obtained from the "zeros" of the absolute values which appear in the addends of the MAD-function.

We next study the MinMax MAD problem on a tree. As usually occurs in this kind of problems, the general strategy is based on solving (independently) a restricted problem on each edge, and then selecting the global solution among all local solutions thus obtained.

3.1.1. Solving the Problem on a Given Edge. For a given edge $e=[u, v]$ with length $\ell_{e}$, we now consider the restricted problem

$$
(\mathrm{MM}-\mathrm{MAD})_{e} \min _{x \in\left[0, \ell_{e}\right]} \max _{t \in\left[t^{-}, t^{+}\right]} F(x, t) .
$$

The main idea for solving it is to identify, for each $x$, a finite set of $t$-values for which the function $F(x, t)$ attains its maximum.

Let $\mathcal{D}_{e}=\left[0, \ell_{e}\right] \times\left[t^{-}, t^{+}\right]$be the feasible domain for this restricted problem. Assuming that each point $x$ in the edge represents the length of the subedge $[u, x]$, then for any vertex $v_{i} \in V, d\left(v_{i}, x\right)=d\left(v_{i}, u\right)+x$ if $u$ belongs to the (unique) path from $v_{i}$ to $x$, otherwise $d\left(v_{i}, x\right)=d\left(v_{i}, u\right)-x$. Equivalently, for all $v_{i} \in V, d\left(v_{i}, x\right)=d\left(v_{i}, u\right)+\delta_{i} x$, with $\delta_{i} \in\{-1,1\}$. Therefore, since $W(t)=A t+B>0, \forall t \in\left[t^{-}, t^{+}\right]$, we can write

$$
\begin{aligned}
F(x, t) & =\frac{1}{W(t)^{2}} \sum_{i=1}^{n} w_{i}(t) \\
& \times\left|\left(d\left(v_{i}, u\right)+\delta_{i} x\right) W(t)-\sum_{i=1}^{n} w_{i}(t)\left(d\left(v_{i}, u\right)+\delta_{i} x\right)\right| .
\end{aligned}
$$

Once each term has been expanded, the function $F(x, t)$ for any $x \in\left[0, \ell_{e}\right]$ can be expressed as follows

$$
\begin{aligned}
F(x, t) & =\frac{1}{W(t)^{2}} \sum_{i=1}^{n} w_{i}(t)\left|a_{i} x t+b_{i} t+c_{i} x+d_{i}\right| \\
& =\frac{1}{W(t)^{2}} \sum_{i=1}^{n} w_{i}(t)\left|f_{i}(x, t)\right|
\end{aligned}
$$

where $f_{i}(x, t)=a_{i} x t+b_{i} t+c_{i} x+d_{i}$, with $a_{i}, b_{i}, c_{i}, d_{i} \in \mathbb{R}$, $\forall i=1, \ldots, n$. 


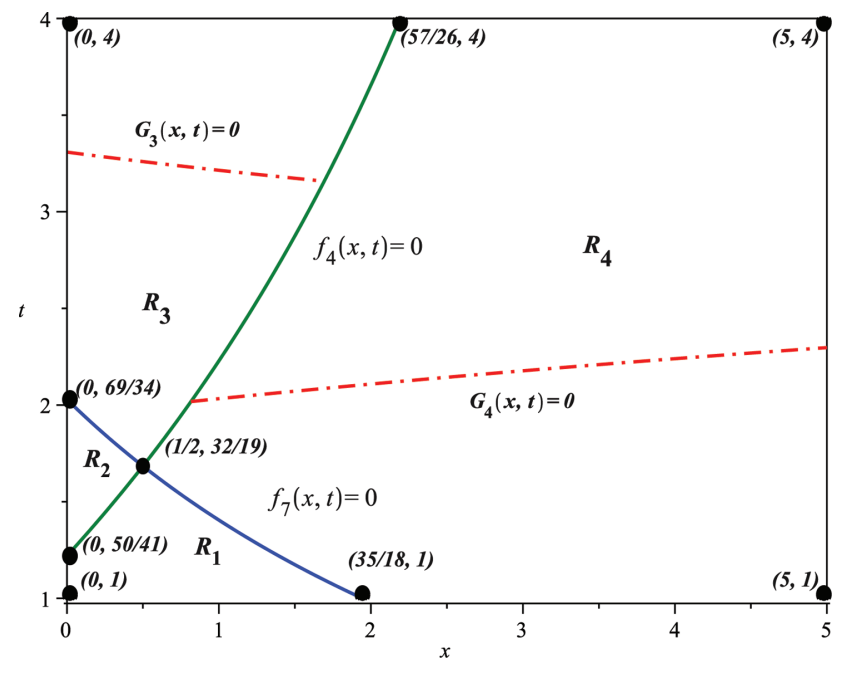

FIG. 2. $\mathcal{P}\left(\mathcal{D}_{e}\right)$ for edge $\left[v_{3}, v_{5}\right]$, with stationary curves $G_{3}(x, t)=0$ and $G_{4}(x, t)=0$. [Color figure can be viewed in the online issue, which is available at wileyonlinelibrary.com.]

The breakpoints of $F(x, t)$ belong to the curves $f_{i}(x, t)=0$, $i=1, \ldots, n$. Each $f_{i}(x, t)=0$ is a hyperbola (a straight line if $a_{i}=0$ ), and it will be called "breakpoint curve." The collection of breakpoint curves $\left\{f_{i}(x, t)=0, i=1, \ldots, n\right\}$ induces a subdivision $\mathcal{P}\left(\mathcal{D}_{e}\right)$ of the feasible domain $\mathcal{D}_{e}$ into a set of full dimensional cells $R_{j}, j=1, \ldots, r_{e}$, such that in the interior of each subregion $R_{j}$, the functions $f_{i}(x, t)$ are not null for $i=1, \ldots, n$. Consequently, $F(x, t)$ is a differentiable function on the interior of each subregion $R_{j}$ of $\mathcal{P}\left(\mathcal{D}_{e}\right)$.

Figure 2 shows the subdivision of the feasible domain for the edge $\left[v_{3}, v_{5}\right]$ of the tree given in Figure 1. In this case there are only two breakpoint curves with nonempty intersection with $\mathcal{D}_{e}=[0,5] \times[1,4]$, obtained from $f_{4}(x, t)=0$ and $f_{7}(x, t)=0$. The remaining hyperbolas lie outside of $\mathcal{D}_{e}$.

Each element $R_{j}$ of the subdivision is a closed and compact set whose boundary may be composed by both several arcs of breakpoint curves ("pieces" of hyperbolas and/or segments) and line segments of the boundary of $\mathcal{D}_{e}$, and whose vertices are the endpoints of such pieces. Let $V\left(\mathcal{P}\left(\mathcal{D}_{e}\right)\right)$ be the set of vertices of the subdivision $\mathcal{P}\left(\mathcal{D}_{e}\right)$. Clearly, the elements of $V\left(\mathcal{P}\left(\mathcal{D}_{e}\right)\right)$ are the vertices of the rectangle $\mathcal{D}_{e}$ together with the intersection points between breakpoints curves as well as between breakpoint curves and the boundary of $\mathcal{D}_{e}$.

For example, Figure 2 shows (with black dots) the vertices of the set $V\left(\mathcal{P}\left(\mathcal{D}_{e}\right)\right)$ for the edge $e=\left[v_{3}, v_{5}\right]$. The breakpoint curves $f_{4}(x, t)=0$ and $f_{7}(x, t)=0$ intersect at the point $\left(\frac{1}{2}, \frac{32}{19}\right)$. Besides, the endpoints of these curves on the boundary of $\mathcal{D}_{e}$ are $\left\{\left(0, \frac{50}{41}\right),\left(\frac{57}{26}, 4\right)\right\}$ and $\left\{\left(0, \frac{69}{34}\right),\left(\frac{35}{18}, 1\right)\right\}$, respectively. All these points together with the vertices of the rectangle $\mathcal{D}_{e},(0,1),(5,1),(0,4),(5,4)$, define the set $V\left(\mathcal{P}\left(\mathcal{D}_{e}\right)\right)$. Moreover, two cells of $\mathcal{P}\left(\mathcal{D}_{e}\right)$ are adjacent if they share a common boundary, which is either a vertex of $\mathcal{P}\left(\mathcal{D}_{e}\right)$ or an arc (or piece) of some breakpoint curve limited by two different vertices of $V\left(\mathcal{P}\left(\mathcal{D}_{e}\right)\right)$, such that it does not contain any vertex of $V\left(\mathcal{P}\left(\mathcal{D}_{e}\right)\right)$ in its interior. In Figure 2, cells $R_{2}$ and $R_{4}$ are adjacent to $R_{3}$ by different pieces of arcs, whereas $R_{1}$ and $R_{3}$ are adjacent by the vertex $(1 / 2,32 / 19)$.

In contrast, for a fixed value $\bar{x} \in\left[0, \ell_{e}\right], F(\bar{x}, t)=$ $\frac{1}{W(t)^{2}} \sum_{i=1}^{n} w_{i}(t)\left|f_{i}(\bar{x}, t)\right|$ is a continuous function of $t$ but in general it is neither concave nor convex on the $t$-interval $\left[t^{-}, t^{+}\right]$. The breakpoints of $F(\bar{x}, t)$ in such interval are obtained from the solutions of $f_{i}(\bar{x}, t)=0, i=1, \ldots, n$. The following result establishes that such breakpoints can not be local maxima of $F(\bar{x}, t)$.

Theorem 4. For a given $\bar{x} \in\left[0, \ell_{e}\right]$, let $t_{i} \in\left(t^{-}, t^{+}\right)$be a breakpoint of $F(\bar{x}, t)$ such that $f_{i}\left(\bar{x}, t_{i}\right)=0$. Then, $t_{i}$ is not a local maximum of $F(\bar{x}, t)$.

Proof. In order to stress the fact that $\bar{x}$ is fixed in this proof, let us denote $F_{\bar{x}}(t):=F(\bar{x}, t)$. The proof is based on studying the left and the right derivatives of $F_{\bar{x}}(t)$ at $t_{i}$. Let us suppose that $f_{j}\left(\bar{x}, t_{i}\right) \neq 0, \forall j \neq i$. The function $F_{\bar{x}}(t)$ can be written as the sum of the following two functions

$$
\begin{aligned}
F_{\bar{x}}(t) & =\frac{1}{W(t)^{2}}\left(\sum_{\substack{j=1 \\
j \neq i}}^{n} w_{j}(t)\left|f_{j}(\bar{x}, t)\right|+w_{i}(t)\left|f_{i}(\bar{x}, t)\right|\right) \\
& =\frac{1}{W(t)^{2}}\left(F_{1, \bar{x}}(t)+F_{2, \bar{x}}(t)\right),
\end{aligned}
$$

where $F_{1, \bar{x}}(t):=\sum_{\substack{j=1 \\ j \neq i}}^{n} w_{j}(t)\left|f_{j}(\bar{x}, t)\right|$ and $F_{2, \bar{x}}(t):=$ $w_{i}(t)\left|f_{i}(\bar{x}, t)\right|$. Let $\mathcal{I} \subset\left[t^{-}, t^{+}\right]$be an open interval (small enough) such that $t_{i} \in \mathcal{I}$ and for $t \in \mathcal{I}, f_{j}(\bar{x}, t) \neq 0$, $\forall j \neq i$. Clearly, $f_{j}(\bar{x}, t)$ has constant sign on $\mathcal{I}$, therefore $\left|f_{j}(\bar{x}, t)\right|=\delta_{j} f_{j}(\bar{x}, t)$, with $\delta_{j} \in\{-1,1\}$. This implies that $F_{1, \bar{x}}(t)$ is a sum of products of two linear functions of $t$, consequently, $F_{1, \bar{x}}(t)$ is a positive parabola on $\mathcal{I}$. Let $s_{1}=F_{1, \bar{x}}^{\prime}\left(t_{i}\right)$ be the slope of $F_{1, \bar{x}}$ at $t_{i}$. On the other hand, $t_{i}$ is a local minimum of $F_{2, \bar{x}}(t)$ (as it is a breakpoint of $\left|f_{i}(\bar{x}, t)\right|$ for which $\left.f_{i}\left(\bar{x}, t_{i}\right)=0\right)$. Without loss of generality we can assume that $t_{i}$ is a root of order 1 of the parabola or straight line $w_{i}(t) f_{i}(\bar{x}, t)$ (since $f_{i}(\bar{x}, t)$ is linear on $t$ and the weights are non-negatives on $\left.\left[t^{-}, t^{+}\right]\right)$. Let $s_{2} \neq 0$ be the slope of $w_{i}(t) f_{i}(\bar{x}, t)$ at $t_{i}$. Since $F_{2, \bar{x}}(t)=w_{i}(t)\left|f_{i}(\bar{x}, t)\right|$, the left and the right derivatives of $F_{2, \bar{x}}(t)$ at $t_{i}$ are $F_{2, \bar{x}}^{\prime}\left(t_{i}^{-}\right)=-\left|s_{2}\right|$ and $F_{2, \bar{x}}^{\prime}\left(t_{i}^{+}\right)=\left|s_{2}\right|$, respectively. Moreover, the derivative of $F_{\bar{x}}(t)$ is given by

$$
\begin{aligned}
F_{\bar{x}}^{\prime}(t)=\frac{1}{W(t)^{2}}\left(-2 A W(t) F_{\bar{x}}(t)+F_{1, \bar{x}}^{\prime}(t)+\right. & \left.F_{2, \bar{x}}^{\prime}(t)\right), \\
& \forall t \in \mathcal{I}, t \neq t_{i} .
\end{aligned}
$$

Let $F_{\bar{x}}^{\prime}\left(t_{i}^{-}\right), F_{\bar{x}}^{\prime}\left(t_{i}^{+}\right)$be the left/right directional derivatives of $F_{\bar{x}}(t)$ at $t_{i}$. By hypothesis, $W(t)=A t+B>0$, for all $t \in\left[t^{-}, t^{+}\right]$, thus we have

$$
\begin{aligned}
& F_{\bar{x}}^{\prime}\left(t_{i}^{-}\right)=\frac{1}{W\left(t_{i}\right)^{2}}\left(Q_{i}+s_{1}-\left|s_{2}\right|\right), \text { and } \\
& F_{\bar{x}}^{\prime}\left(t_{i}^{+}\right)=\frac{1}{W\left(t_{i}\right)^{2}}\left(Q_{i}+s_{1}+\left|s_{2}\right|\right)
\end{aligned}
$$


with $Q_{i}=-2 A W\left(t_{i}\right) F_{\bar{x}}\left(t_{i}\right)$. Clearly, $F_{\bar{x}}^{\prime}\left(t_{i}^{-}\right)<F_{\bar{x}}^{\prime}\left(t_{i}^{+}\right)$which implies that $F_{\bar{x}}(t)$ can not have a local maximum at $t_{i}$. This concludes the proof.

Note that the proof would be similar if there were more functions which canceled out at $t_{i}$. Indeed, let $I_{\bar{x}} \subseteq\{1, \ldots, n\}$ be the set of subindices such that $f_{j}\left(\bar{x}, t_{i}\right)=0, \forall j \in I_{\bar{x}}$, and $f_{j}\left(\bar{x}, t_{i}\right) \neq 0, \forall j \notin I_{\bar{x}}$. In this case, $F_{\bar{x}}(t)$ decomposes as above, but now $F_{1, \bar{x}}(t)=\sum_{\substack{j \neq 1 \\ j \neq l_{\bar{x}}}}^{n} w_{j}(t)\left|f_{j}(\bar{x}, t)\right|$ and $F_{2, \bar{x}}(t)=\sum_{j \in I_{\bar{x}}} w_{j}(t)\left|f_{j}(\bar{x}, t)\right|$.

This result implies that, for each $x$, the local maxima of $F(x, t)$ do not lie on the relative interior of any breakpoint curve.

Corollary 5. For each $x \in\left[0, \ell_{e}\right]$, the solutions of $\max _{t} F(x, t)$ are attained at either an interior point of a cell $R_{j}$ for some $j=1, \ldots, r_{e}$ or at a point of the boundary of $\mathcal{D}_{e}$.

Let $\operatorname{int}\left(R_{j}\right)$ denote the interior of $R_{j}$. We now study the solutions of $\max _{t}\{F(x, t)\}$ on each cell $R_{j}$. Note that in each $R_{j}$, the following facts hold:

1. All functions $f_{i}(x, t), i=1, \ldots, n$ have constant sign. Therefore, $\forall i=1, \ldots, n$ we can write $\left|f_{i}(x, t)\right|=$ $\delta_{i} f_{i}(x, t), \forall(x, t) \in R_{j}$.

2. Consequently, the objective function $F(x, t)$ can be expressed as follows

$$
\begin{aligned}
F(x, t) & =F_{j}(x, t) \\
& =\frac{P_{j 1}(x) t^{2}+P_{j 2}(x) t+P_{j 3}(x)}{(A t+B)^{2}}, \quad \forall(x, t) \in R_{j}
\end{aligned}
$$

where each $P_{j i}(x)$ is a linear function, for $i=1,2,3$.

In the interior of each $R_{j}$, for a fixed $x, F_{j}(x, t)$ is a continuous and differentiable function with at most one critical point and two additional candidates for maximum if the boundary of $R_{j}$ has not empty intersection with $t=t^{-}$and $t=t^{+}$. For each $x$-value such that the vertical straight line at $x$ crosses $\operatorname{int}\left(R_{j}\right)$, if the maximum $t$-value which solves $\max _{t \in\left[t^{-}, t^{+}\right]}\left\{F_{j}(x, t)\right\}$ belongs to int $\left(R_{j}\right)$ then it should belong to the $j$-th curve of stationary points $G_{j}(x, t)=0$ (or $j$-th stationary curve), given by

$$
G_{j}(x, t)=\frac{\partial F_{j}(x, t)}{\partial t}=\frac{\widehat{P}_{j 1}(x) t+\widehat{P}_{j 2}(x)}{(A t+B)^{3}}=0
$$

where $\widehat{P}_{j 1}(x)=2 B P_{j 1}(x)-A P_{j 2}(x)$, and $\widehat{P}_{j 2}(x)=B P_{j 2}(x)-$ $2 A P_{j 3}(x)$. This expression implies that there exists (at most) one stationary curve on each cell of the subdivision. In the example illustrated in Figure 2 we have only two stationary curves: $G_{3}(x, t)=0$ and $G_{4}(x, t)=0$ (drawn in dashed lines), because the remaining ones are out of the feasible domain.

By extending this analysis to all cells of $\mathcal{P}\left(\mathcal{D}_{e}\right)$, Corollary 5 can be rewritten as follows:
Property 6. A solution of $\min _{x \in e} \max _{t \in\left[t^{-}, t^{+}\right]}\{F(x, t)\}$ is either a point of some stationary curve $G_{j}(x, t)=0, j=$ $1, \ldots, r_{e}$, or a point in one of the two extreme $t$-segments $t=t^{-}$and $t=t^{+}$of $\mathcal{D}_{e}$.

Clearly, both $F\left(x, t^{-}\right)$and $F\left(x, t^{+}\right)$are functions of $x$. In contrast, when $(x, t)$ moves over a stationary curve $G_{j}(x, t)=$ 0 the function $F(x, t)$ can be expressed as a function of $x$ by isolating (if possible) $t=g_{j}(x)$ from $G_{j}(x, t)=0$ and replacing it in the objective function. Let $I_{j}$ be the projection of $G_{j}(x, t)=0$ over the $x$-edge. Therefore

$$
\begin{aligned}
(x, t) \in G_{j}(x, t)=0 \Longrightarrow & F_{j}(x, t)=F\left(x, g_{j}(x)\right), \\
& \quad \text { with } G_{j}\left(x, g_{j}(x)\right)=0, \text { for } x \in I_{j} .
\end{aligned}
$$

If it is not possible to isolate $t$ from $G_{j}(x, t)=0$, which happens when $\widehat{P}_{j 1}(x)=0$, then the stationary curve has the form $x=x^{e, j}$ where $x^{e, j}$ is a constant. In this case, the maximum on $R_{j}$ is attained at a single point $\left(x^{e, j}, t^{e, j}\right)$, that is, $F\left(x^{e, j}, t^{e, j}\right)=\max _{t}\left\{F\left(x^{e, j}, t\right):\left(x^{j}, t\right) \in R_{j}\right\}$. For the sake of simplicity, we maintain the notation $g_{j}(x)=t^{e, j}$ and $I_{j}=\left\{x^{e, j}\right\}$ for this case.

Note that after substituting $t=g_{j}(x), F\left(x, g_{j}(x)\right)$ can be expressed as $\frac{1}{W(t)^{2}} \frac{Q_{j_{1}}^{(3)}(x)}{Q_{j_{2}}^{(2)}(x)}$, where $Q_{j_{1}}^{(3)}(x), Q_{j_{2}}^{(2)}(x)$ are polynomials in $x$, and the superindex (3) and (2) indicate the maximum degree of each polynomial.

From the above argument, the collection of all the functions $\left\{F\left(x, g_{j}(x)\right), j=1, \ldots, r_{e}\right\}$ together with $F\left(x, t^{-}\right)$, $F\left(x, t^{+}\right)$provides the objective value over the stationary curves and the extreme $t$-segments.

Theorem 7. The solution of $\min _{x \in e} \max _{t \in\left[t^{-}, t^{+}\right]} F(x, t)$ is the pointwise minimum of the upper envelope $U_{e}(x)$ of the collection of $r_{e}+2$ functions

$$
\left\{\left\{F\left(x, g_{j}(x)\right), x \in I_{j}, j=1, \ldots, r_{e}\right\}, F\left(x, t^{-}\right), F\left(x, t^{+}\right)\right\}
$$

where $r_{e}$ is the number of cells of $\mathcal{P}\left(\mathcal{D}_{e}\right)$ of the feasible domain.

In conclusion, the main procedure for solving the restricted problem on a given edge $e$ involves the following steps:

Step 1: Obtain the $O(n)$ breakpoint curves (in $O(n)$ time).

Step 2: Construct $\mathcal{P}\left(\mathcal{D}_{e}\right)$, i.e. the arrangement of the breakpoint curves (hyperbolas). The arrangement of $n$ hyperbolas in the plane can be done in $O\left(n^{2} \alpha(n)\right)$ time and $O\left(n^{2}\right)$ space (see Edelsbrunner et al. [18], McKenna and O'Rourke [21]), where $\alpha(n)$ is the inverse of the Ackermann function, see Appendix. On the other hand, since each two breakpoint curves intersect in at most two points, the subdivision $\mathcal{P}\left(\mathcal{D}_{e}\right)$ has $O\left(n^{2}\right)$ vertices and $O\left(n^{2}\right)$ cells. Note that the closed form of the objective function $F(x, t)$ in each cell, as a continuous and differentiable function, 
can be computed by updating from one cell of the arrangement to any adjacent one in constant time. Indeed, there are a fixed number of functions $f_{i}(x, t)$ with $i=1, \ldots, n$, changing their sign from one cell to any adjacent one. In particular, this number is given by the number breakpoint curves that are common boundary of the two cells (in most cases only one). Thus, this computation does not increment the overall complexity.

Step 3: Apply Theorem 7, which requires to construct the upper envelope $U_{e}(x)$ of the collection of $r_{e}+2$ functions $\left\{F\left(x, g_{j}(x)\right), j=1, \ldots, r_{e}, F\left(x, t^{-}\right), F\left(x, t^{+}\right)\right\}$. On each $R_{j}$, the stationary curve $G_{j}(x, t)=0$ and the the subsequent function $F\left(x, g_{j}(x)\right)$ is obtained in constant time. The functions $F\left(x, g_{j}(x)\right)$ could be partially defined over the $x$-interval $\left[0, \ell_{e}\right]$, therefore they must be considered as arcs. Moreover, as such functions are the product of $W(t)^{-2}$ and the quotient of two polynomials with maximum degree bounded above by three and two, respectively, then the number of intersection points between any pair of them is at most five. By [29, Theorem 6.1], the upper envelope of these functions can be computed in $O\left(\lambda_{6}\left(r_{e}+2\right) \log \left(r_{e}+2\right)\right)$ time, and the complexity of computing the minimum on $x$ is dominated by the number of transition points, $O\left(\lambda_{7}\left(r_{e}+2\right)\right)$, on the upper envelope (see Appendix for further details).

Because the complexity of computing the upper envelope (Step 3) dominates the complexity of Step 1 and 2 and $r_{e}+2=O\left(n^{2}\right)$, we conclude that the restricted problem $(\mathrm{MM}-\mathrm{MAD})_{e}$ can be solved in $O\left(\lambda_{6}\left(n^{2}\right) \log n\right)$ time, which gives a complexity of $O\left(n \lambda_{6}\left(n^{2}\right) \log n\right)$ for solving the problem over the overall tree.

\subsection{The Problem on a Network}

We now consider the (MM-MAD) problem on a general network $N(V, E)$. In this case, each edge of the network is partitioned in several subedges such that on each subedge all distance functions are linear. Therefore, the global problem is treated by solving a collection of independent optimization subproblems obtained by restricting the problem to each subedge of the partition.

Let $e=[u, v]$ be a given edge of the network. For each $v_{i} \in V$, the distance function $d\left(v_{i}, x\right)$ is piecewise linear and concave in $e$ with at most one maximum $\bar{x}_{i}$ for which $d\left(v_{i}, x\right)$ is the same via vertex $u$ as via vertex $v$. This point (if any) is called "edge bottleneck point" with respect to vertex $v_{i}$ (see Church and Garfinkel [15]). Henceforth we will say bottleneck point instead of edge bottleneck point. Each edge has $O(n)$ bottleneck points, which can be computed and sorted in $O(n \log n)$ time. These points together with the end vertices $u$ and $v$ determine a partition of the edge $e$, and each closed subedge $\left[\bar{x}_{i}, \bar{x}_{i+1}\right]$ delimited by two adjacent points of this partition is called a "primary region". Note that on each primary region the behavior and properties of the involved functions is the same as on each edge of a tree. For example, for a fixed $t$-value, $F(x, t)$ is a convex, piecewise linear function on each primary region on the edge (although in general $F(x, t)$, as function of $x$, is neither concave nor convex on the overall edge).

Therefore, on each primary region we consider the restricted subproblem

$$
\min _{x \in\left[\bar{x}_{i}, \bar{x}_{i+1}\right]} \max _{t \in\left[t^{-}, t^{+}\right]} F(x, t),
$$

which can be solved in $O\left(\lambda_{6}\left(n^{2}\right) \log n\right)$ time by applying the procedure described in the previous subsection. Because there are $O(m n)$ primary regions on the network, the final complexity is $O\left(m n \lambda_{6}\left(n^{2}\right) \log n\right)$.

\section{THE MINMAX REGRET MEAN ABSOLUTE DEVIATION PROBLEM}

We now consider the (MMR-MAD) location problem, i.e.,

(MMR-MAD) $\min _{x \in N} \max _{t \in\left[t^{-}, t^{+}\right]}\left\{F(x, t)-F^{*}(t)\right\}$,

$$
\text { with } F^{*}(t)=\min _{x \in N} F(x, t)
$$

As in the previous section, we first study the problem on a tree network $T(V, E)$.

\subsection{Solving the Problem on a Tree Network}

On a tree network, $T=(V, E)$, the solution strategy is again based on solving the problem restricted to each edge of the tree. Thus, for a given edge $\bar{e}=[u, v]$ with length $\ell_{\bar{e}}$, let us consider the restricted problem

$(\mathrm{MMR}-\mathrm{MAD})_{\bar{e}} \min _{x \in\left[0, \ell_{\bar{e}}\right]} \max _{t \in\left[t^{-}, t^{+}\right]}\left\{F(x, t)-F^{*}(t)\right\}$

$$
\text { with } F^{*}(t)=\min _{x \in T} F(x, t)
$$

4.1.1. Computing $F^{*}(t)$ First of all, we give a procedure for computing the function $F^{*}(t)$. It is known (Section 3.1) that for each $t, F(x, t)$ is a piecewise linear and convex function of $x \in\left[0, \ell_{e}\right]$ for any $e \in E$, and its breakpoints belong to the breakpoint curves. This means that, for each $t$, the minimum $F^{*}(t)$ is attained at either some breakpoint curve of $\mathcal{P}\left(\mathcal{D}_{e}\right)$ or at an extreme segment $x=0, x=\ell_{e}$ of the feasible domain $\mathcal{D}_{e}=\left[0, \ell_{e}\right] \times\left[t^{-}, t^{+}\right]$for some $e \in E$.

To obtain domains where $F^{*}(t)$ has a differentiable expression, we consider the projections over the $t$-edge of $V(\mathcal{P}(\mathcal{D})):=\bigcup_{e \in E} V\left(\mathcal{P}\left(\mathcal{D}_{e}\right)\right)$ (recall that $V\left(\mathcal{P}\left(\mathcal{D}_{e}\right)\right)$ is the set of vertices of the subdivision $\left.\mathcal{P}\left(\mathcal{D}_{e}\right)\right)$. From these points, we define the list $\Sigma=\left\{t_{1}, \ldots, t_{\sigma}\right\}$ of $t$-points, where $t^{-}=t_{1}$, $t^{+}=t_{\sigma}$ and $t_{k} \leq t_{k+1}$ for any $k=1, \ldots, \sigma-1$. Observe that $\sigma=O\left(n^{3}\right)$.

In contrast, when $(x, t) \in e \times\left[t_{k}, t_{k+1}\right]$, with $k=$ $1, \ldots, \sigma-1$, moves along a piece of breakpoint curve

$$
f_{e, i}(x, t):=a_{e, i} x t+b_{e, i} x+c_{e, i} t+d_{e, i}=0,
$$



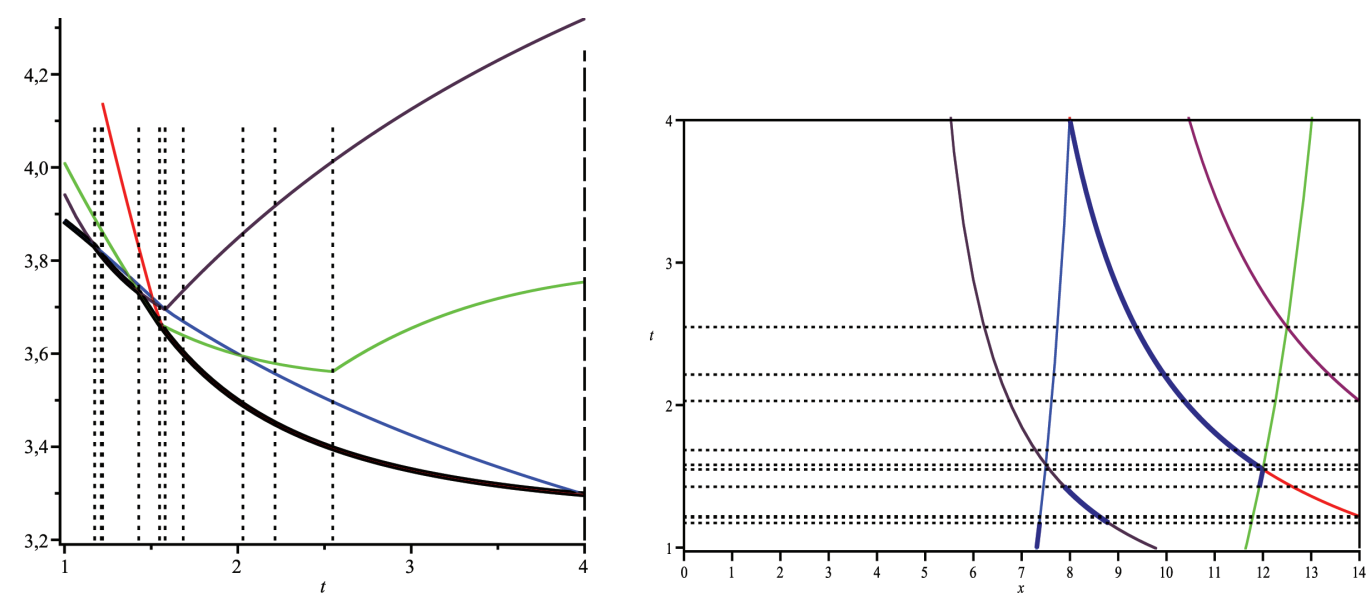

FIG. 3. $L(t)$ (left), and breakpoint curves providing $F^{*}(t)$ (right). [Color figure can be viewed in the online issue, which is available at wileyonlinelibrary.com.]

$F(x, t)$ can be expressed as a function of $t$ by isolating (if possible) $x=\widehat{f}_{e, i}(t)$ from $f_{e, i}(x, t)=0$. Let $\widehat{I}_{e, i}$ be the projection of $f_{e, i}(x, t)=0$ over the $t$-edge. Therefore, when $t \in \widehat{I}_{e, i}$ and $x$ satisfies $f_{e, i}(x, t)=0$, we have $\left.F(x, t)=F \widehat{(}_{e, i}(t), t\right)$. The case in which it is not possible to isolate $x$ from $f_{e, i}(x, t)=0$ means that the equation $f_{e, i}(x, t)=0$ does not depend on $x$. Hence, $f_{e, i}(x, t)$ is a linear function of $t$, it has the form $t=t^{e, i}$, where $t^{e, i}$ is a constant. In this case, the optimal value $F^{*}\left(t^{e, i}\right)$ is attained at a single point $\left(x^{e, i}, t^{e, i}\right)$ (which can be obtained in $O(n)$ time, Mesa et al. [23]). For the sake of simplicity, we maintain the notation $\widehat{f}_{e, i}(t)=x^{e, i}$ and $\widehat{I}_{e, i}=\left\{t^{e, i}\right\}$ for this case.

By construction, the lower envelope of the collection of all these functions gives, at each $t$, the function $F^{*}(t)$.

Property 8. The function $F^{*}(t)$ is obtained from the lower envelope $L(t)$ of the collection

$$
\begin{aligned}
\left\{\left\{F\left(\widehat{f}_{e, i}(t), t\right), t\right.\right. & \left.\in \widehat{I}_{e, i}, i=1, \ldots, n, F(0, t), F\left(\ell_{e}, t\right)\right\}, \\
e \in E, t & \left.\in\left[t_{k}, t_{k+1}\right], k=1, \ldots, \sigma-1\right\} .
\end{aligned}
$$

This collection has at most $(\sigma-1)(n+2)(n-1)=O\left(n^{5}\right)$ functions, which can be partially defined on the interval $\left[t^{-}, t^{+}\right]$, and each function is given by the factor $W(t)^{-2}$ multiplied by the quotient of two polynomials with maximum degree bounded above by 3 and 1, respectively, then the number of intersection points between any pair of functions in the collection (2) in its corresponding $t$-interval is at most four. By Theorem 6.5 in Sharir and Agarwal [29] (see Appendix), the complexity of $L(t)$ is bounded by $O\left(\lambda_{6}\left(n^{5}\right)\right)$ and it can be computed in $O\left(\lambda_{5}\left(n^{5}\right) \log n\right)$.

The lower envelope is described by a connected sequence of arcs (ordered from left to right). Let $\tau=\left\{t_{1}, \ldots, t_{g}\right\}$ be the breakpoints of $L(t)$, with $g=O\left(\lambda_{6}(M 5)\right)$. By construction, $\tau$ contains the set $\Sigma$ together with the intersection points of those functions of collection (2) which appear in the envelope. Moreover, ach arc composing the envelope $L(t)$ is a function of the collection (2). Because each function of such collection is obtained from the objective function over some piece of breakpoint curve (or over an extreme $x$-value), we have the following result.

Lemma 9. Let $t_{k}, t_{k+1}$ be two consecutive breakpoints of $L(t)$ with $k=1, \ldots, g-1$. For all $\bar{t} \in\left[t_{k}, t_{k+1}\right], F^{*}(\bar{t})$ is attained over either the same piece of breakpoint curve or the same extreme segment $x=0, x=\ell_{e}$ of $\mathcal{D}_{e}$, for some edge $e \in E$.

Figure 3 (left) shows the lower envelope $L(t)$ corresponding to the tree of Figure 1. In this particular example, it happens that for all $t \in\left[t^{-}, t^{+}\right]$, all the functions $F\left(\widehat{(f e}_{e, i}(t), t\right)$ composing this envelope move over breakpoint curves which belong to the same edge $e=\left[v_{2}, v_{3}\right]$ (with length 14). (Note that in general different pieces of this lower envelope $L(t)$ may belong to breakpoint curves in different edges.)

In Example 3, the set $V(\mathcal{P}(\mathcal{D}))$ contains the points $(15 / 2,49 / 31),(12,48 / 31),(25 / 2,135 / 53)$ (obtained from $\left.\left[v_{2}, v_{3}\right]\right),(0,50 / 41),(0,69 / 34)$ (obtained from $\left.\left[v_{3}, v_{4}\right]\right)$, $(12,31 / 14)$ and $(6,40 / 33)$ (obtained from $\left[v_{5}, v_{6}\right]$ and $\left[v_{5}, v_{7}\right]$, respectively), $(1 / 2,32 / 19),(0,69 / 34),(0,50 / 41)$ (obtained from $\left[v_{2}, v_{3}\right]$ ), and eventually some others that will project onto $t^{-}=1$ or $t^{+}=4$ that can be omitted because these elements are always included in $\tau$. The list $\Sigma$ contains the ordinates of these points, and the list $\tau$ of the breakpoints of $L(t)$ is given by

$$
\tau=\left\{1,1.173, \frac{40}{33}, \frac{50}{41}, 1.427, \frac{48}{31}, \frac{49}{31}, \frac{32}{19}, \frac{69}{34}, \frac{31}{14}, \frac{135}{53}, 4\right\} .
$$

Figure 3 (right) displays all the breakpoint curves of this edge and, in thicker lines, it also shows the pieces of these breakpoint curves corresponding to the respective arcs of $L(t)$ over which $F^{*}(t)$ is attained. For example, in the first $t$-subinterval $[1,1.173]$ of the left picture, the right picture shows that $F^{*}(t)$ is attained over the first piece $f_{e, 1}(x, t)=0$, and so on. 
4.1.2. Solving (MMR-MAD) $)_{\bar{e}}$ The list $\tau$ defined in the previous subsection induces a new subdivision of the feasible domain $\mathcal{D}_{\bar{e}}$ obtained when the cells $\left\{R_{j}, j=1, \ldots, r_{\bar{e}}\right\}$ in $\mathcal{P}\left(\mathcal{D}_{\bar{e}}\right)$ are crossed by the set of lines $t=t_{k}, \forall t_{k} \in \tau$. Let $\mathcal{P}^{\prime}\left(\mathcal{D}_{\bar{e}}\right)$ be this new subdivision, in which each $R_{j}$ is subdivided into a set of smaller cells, and let $\left\{R_{s}^{\prime}, s=1, \ldots, r_{\bar{e}}^{\prime}\right\}$ be the set of cells of $\mathcal{P}^{\prime}\left(\mathcal{D}_{\bar{e}}\right)$. By construction, each cell of $\mathcal{P}^{\prime}\left(\mathcal{D}_{\bar{e}}\right)$ is a subset of (or coincides with) a cell of $\mathcal{P}\left(\mathcal{D}_{\bar{e}}\right)$.

Figure 4 shows, for the edge $\bar{e}=\left[v_{3}, v_{5}\right]$, the subdivision $\mathcal{P}^{\prime}\left(\mathcal{D}_{\bar{e}}\right)$ obtained from the above set $\tau$. The points of $\tau$ define the horizontal dashed lines displayed in this figure.

The following facts hold:

1. The function $F^{*}(t)$ is differentiable on $\left(t_{k}, t_{k+1}\right)$, for $k=$ $1, \ldots, g-1$.

2. In the interior of each cell of $\mathcal{P}^{\prime}\left(\mathcal{D}_{\bar{e}}\right), F(x, t)-F^{*}(t)$ is a differentiable function, and $F^{*}(t)$ is attained over the same piece of breakpoint curve $f_{e, i}(x, t)=0$, (for some $e \in E)$.

These properties of the objective function on each $R_{s}^{\prime}$ provide similar conditions to those established for the problem analyzed in Section 3.1.1 (Theorem 4 is still valid for $F(x, t)-F^{*}(t)$ with $\left.t \in\left(t_{k}, t_{k+1}\right), k=1, \ldots, g-1\right)$. Thus, we will apply the same strategy as in that case. This is based on identifying on each subregion the set of points where the maximum on $t$ is attained, and then solving the restricted problem by finding the minimum over all set of points obtained from all subregions.

In each subregion $R_{s}^{\prime}$ of $\mathcal{P}^{\prime}\left(\mathcal{D}_{\bar{e}}\right)$, the solutions of $\max _{t}\left\{F(x, t)-F^{*}(t):(x, t) \in R_{s}^{\prime}\right\}$ satisfy

$$
H_{s}(x, t)=\frac{\partial\left(F(x, t)-F^{*}(t)\right)}{\partial t}=0
$$

Let $J_{s}$ be the projection of $H_{s}(x, t)=0$ over the $x$-edge, and $t=h_{s}(x)$ be the function of $x$ obtained by isolating (if possible) $t$ from such equation. Because $\mathcal{P}^{\prime}\left(\mathcal{D}_{\bar{e}}\right)$ is finer than $\mathcal{P}\left(\mathcal{D}_{\bar{e}}\right)$ and $R_{s}^{\prime} \subseteq R_{j}$, for some cell $R_{j} \in \mathcal{P}\left(\mathcal{D}_{\bar{e}}\right)$, then $\frac{\partial F(x, t)}{\partial t}=G_{j}(x, t)$. In contrast, $F^{*}(t)=F\left(\widehat{f}_{e, i}(t), t\right)$ for some $i=1, \ldots, n$, therefore $\frac{\partial F\left(\widehat{(f e, i}_{e, t}(t), t\right)}{\partial t}=\frac{1}{W(t)^{3}} \frac{B_{e, i s}^{(3)}(t)}{\left(a_{e, i}^{s} t+c_{e, i}^{s}\right)^{2}}$, where $B_{e, i, s}^{(3)}(t)$ is a polynomial in $t$ of maximum degree 3 , and $a_{e, i}^{s} t+$ $c_{e, i}^{s,} \neq 0$ (the case $a_{e, i}^{s} t+c_{e, i}^{s}=0$ corresponds to $F^{*}(t)=$ $F^{*}\left(t^{e, i, s}\right)$, thus $\left.\frac{\partial\left(F(x, t)-F^{*}(t)\right)}{\partial t}=\frac{\partial F(x, t)}{\partial t}\right)$. Therefore, $H_{s}(x, t)=$ 0 is a bivariate polynomial in $x, t$ of maximum degree 3 in $t$ and 1 in $x$. This implies that one can always obtain $t=$ $h_{s}(x)$ as a function of $x$ by using the closed expression of the solutions of the equation of 3rd degree. Over each curve $H_{s}(x, t)=0$ the objective function $F(x, t)-F^{*}(t)$ can be expressed as a function of $x$ in the following way

$$
F\left(x, h_{s}(x)\right)-F^{*}\left(h_{s}(x)\right), \text { for } x \in J_{s} .
$$

If it is not possible to isolate $t$ from $H_{s}(x, t)=0$, then the curve $H_{s}(x, t)=0$ can be expressed as $x=$ $x^{s}$, where $x^{s}$ is a constant. In this case, the solution of $\max _{t}\left\{F\left(x^{s}, t\right)-F^{*}(t):\left(x^{s}, t\right) \in R_{s}^{\prime}\right\}$ is attained at a single

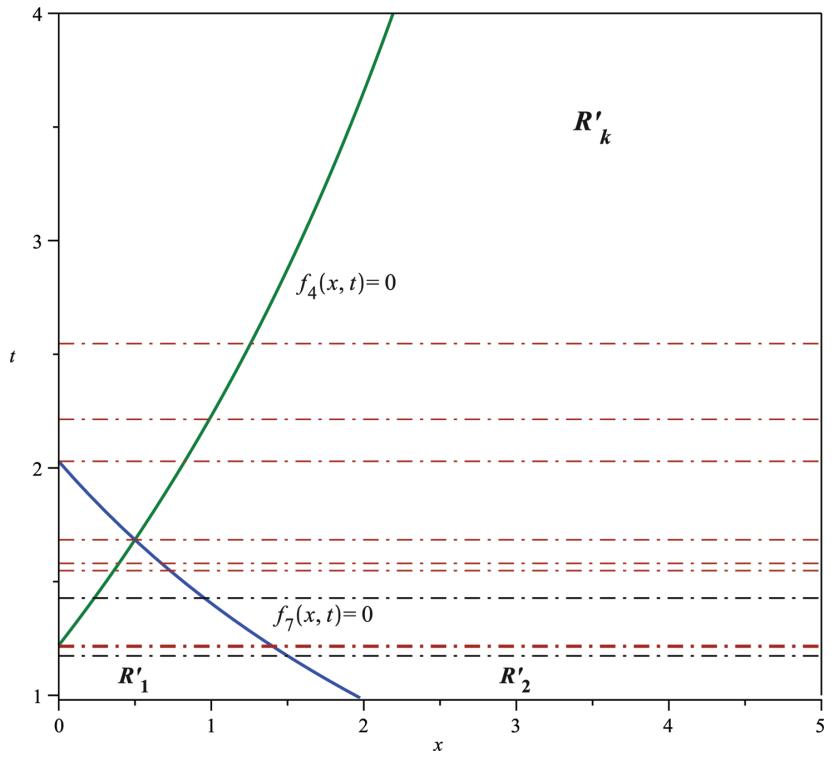

FIG. 4. New subdivision $\mathcal{P}^{\prime}\left(\mathcal{D}_{\bar{e}}\right)$, and cells $R_{k}^{\prime}$, for edge $\bar{e}=\left[v_{3}, v_{5}\right]$. [Color figure can be viewed in the online issue, which is available at wileyonlinelibrary.com.]

point $\left(x^{s}, t^{s}\right)$. For simplicity, we also maintain the notation for this case, that is, $h_{s}(x)=t^{s}$ and $J_{s}=\left\{x^{s}\right\}$.

The function $F\left(x, h_{s}(x)\right)-F^{*}\left(h_{s}(x)\right)$, for $x \in J_{s}$ provides the objective value over the curve $H_{s}(x, t)=0$ of stationary points in each cell $R_{s}^{\prime}$. Therefore the problem $\max _{t \in\left[t^{-}, t^{+}\right]}\left\{F(x, t)-F^{*}(t)\right\}$ can be expressed as the upper envelope $U_{\bar{e}}^{\prime}(x)$ of the following collections of functions $\left\{F\left(x, h_{s}(x)\right)-F^{*}\left(h_{s}(x)\right), x \in J_{s}(x)\right\}$ over all cells of the subdivision of the feasible domain. Consequently, the solution of the restricted problem can be established as follows:

Theorem 10. The solution of Problem (MMR-MAD) $)_{\bar{e}}$ : $\min _{x \in\left[0, \ell_{\bar{e}}\right]} \max _{t \in\left[t^{-}, t^{+}\right]}\left\{F(x, t)-F^{*}(t)\right\}$ is the pointwise minimum of the upper envelope $U_{\bar{e}}^{\prime}(x)$ of the collection of functions

$$
\begin{aligned}
\left\{F\left(x, h_{s}(x)\right)-F^{*}\left(h_{s}(x)\right), x \in J_{s}(x), s=1, \ldots, r_{\bar{e}}^{\prime},\right. \\
\left.F\left(x, t_{k}\right)-F^{*}\left(t_{k}\right), k=1, \ldots, g\right\}
\end{aligned}
$$

where $r_{\bar{e}}^{\prime}$ is the number of subregions of $\mathcal{P}^{\prime}\left(\mathcal{D}_{\bar{e}}\right)$.

From Section 3.1.1, we can assume that in a preprocessing phase, the arrangement of the $n$ breakpoint curves together with the computation of the closed form of the objective function as a continuous and differentiable function on each cell of the initial subdivision $P\left(\mathcal{D}_{\bar{e}}\right)$ is already obtained in $O\left(n^{2} \alpha(n)\right)$. Besides, we note in passing that the maximum number of intersections of any two curves of the above family (3) is bounded above by a constant which does not depend on $n$. Then, to discuss the complexity of solving Problem $(\text { MMR-MAD) })_{\bar{e}}$, it is necessary to analyze each phase in the procedure below: 
Phase 1: Compute $F^{*}(t)$, given by the lower envelope $L(t)$. As we have already pointed out, $L(t)$ can be constructed in $O\left(\lambda_{5}\left(n^{5}\right) \log n\right)$ time, and its complexity is $O\left(\lambda_{6}\left(n^{5}\right)\right.$ (Sharir and Agarwal [29], Pach and Sharir [26]).

Phase 2: Obtain the subdivision $\mathcal{P}^{\prime}\left(\mathcal{D}_{\bar{e}}\right)$. This is done by intersecting the $\tau$-partition with the $O\left(n^{2}\right)$ cells of $\mathcal{P}\left(\mathcal{D}_{\bar{e}}\right)$ obtained from the preprocessing phase. The cardinality of $\tau$ is $g=O\left(\lambda_{6}\left(n^{5}\right)\right)$, and the subdivision $\mathcal{P}^{\prime}\left(\mathcal{D}_{\bar{e}}\right)$ has $O\left(n^{2} \lambda_{6}\left(n^{5}\right)\right)$ cells, which is the complexity of $r_{\bar{e}}^{\prime}$.

Phase 3: Construct $U_{\bar{e}}^{\prime}(x)$, the upper envelope of the collection of $O\left(n^{2} \lambda_{6}\left(n^{5}\right)\right)$ (partially defined) curves described in Theorem 10. This can be done in $O\left(n^{2} \lambda_{6}\left(n^{5}\right) \log ^{*} n \log n\right)$ time, see Appendix.

This last complexity dominates the previous ones, therefore solving the (MMR-MAD) $)_{\bar{e}}$ problem requires $O\left(n^{2} \lambda_{6}\left(n^{5}\right) \log ^{*} n \log n\right)$ time, which implies that the complexity for solving the problem over the tree is $O\left(n^{3} \lambda_{6}\left(n^{5}\right) \log ^{*} n \log n\right)$.

\subsection{The Problem on a Network}

As in the previous section, on a general network the problem is analyzed by applying the above procedure for solving the problem restricted to each primary region of the network. The complexity of each restricted problem dominates the preprocessing phase (for computing the distance matrix), consequently the overall complexity is $O\left(m n^{3} \lambda_{6}\left(n^{5}\right) \log ^{*} n \log n\right)$. We would like to remark that this complexity is higher than $O\left(n^{9}\right)$. This high complexity may suggest to consider alternative approaches to analyze these problems which may lead to better complexity bounds.

\section{CONCLUDING REMARKS}

In this article, we considered two robust versions of the mean absolute deviation problem on networks with dynamic evolution of node weights, which are modeled by means of linear functions of a parameter. For each robust criteria, we have studied the corresponding optimization problem on both tree networks and cyclic networks, we have provided exact algorithms for solving them and discussed their corresponding complexities.

As pointed out by Erkut [12], direct minimization of typical inequality measures (including MAD) contradicts the minimization of individual outcomes, and the facility located at (or near) infinity will provide (almost) perfectly equal service (in fact, rather lack of service) to all the clients. Nevertheless, some absolute inequality measures like MAD can be combined with the mean itself into optimization criteria that remain in harmony with both inequality minimization and minimization of distances (Ogryczak [24] and references therein). The results in this article allow to tackle with the same tools the minimization of the combined median and MAD objective function $M(x, t)+\alpha F(x, t)$ with $\alpha>0$ which guarantees equitable minimization of distances. The reader may note that using the same notation as in (1), we obtain the following representation:

$$
\begin{aligned}
M(x, t)+\alpha F(x, t)= & \frac{1}{W(t)} \sum_{i=1}^{n} w_{i}(t) d\left(v_{i}, x\right) \\
& +\alpha \frac{1}{W(t)} \sum_{i=1}^{n} w_{i}(t)\left|d\left(v_{i}, x\right)-M(x, t)\right| \\
= & \frac{1}{W(t)^{2}} \sum_{i=1}^{n} w_{i}(t)\left(\left(d\left(v_{i}, u\right)\right.\right. \\
& \left.\left.+\delta_{i} x\right) W(t)+\alpha\left|f_{i}(x, t)\right|\right) .
\end{aligned}
$$

From this expression, we can reproduce a similar argument as the one done in the article for the function $F(x, t)$.

Different open problems arise when edge lengths can change dynamically with the parameter and also if one considers alternative functions for describing the dynamic nature of the parameter. For example, in certain periods of time, the evolution of some populations can be better represented by piecewise linear or polynomial functions than by linear ones. Also, modeling the deviation from a nominal value of the weights may require more complex functions than linear. However, these problems clearly lie out of the scope of this article and therefore, they are left for a follow up article.

\section{APPENDIX}

In this Appendix, we include, for the sake of completeness and readability, an important result that is used in the article.

Theorem 11 (Sharir and Agarwal (1995), Theorem 6.5). Given a set of $n x_{1}$-monotone Jordan arcs with at most $s$ intersections between any pair of arcs, its lower envelope has an $O\left(\lambda_{s+2}(n)\right)$ complexity, and it can be computed in $O\left(\lambda_{s+1}(n) \log n\right)$ time.

$\lambda_{s}(n)$ is the maximum length of a Davenport-Schinzel sequence of order $s$ on $n$ symbols. The reader is referred to Chapter 3 in Sharir and Agarwal (1995) for the exact definitions and properties of the functions $\lambda_{s}(n)$. We note that $\lambda_{1}(n)=O(n), \lambda_{2}(n)=O(n), \lambda_{3}(n)=\theta(n \alpha(n))$, and $\lambda_{4}(n)=\theta\left(n 2^{\alpha(n)}\right)$, where $\alpha(n)$ is the inverse of the Ackermann function which grows very slowly. Observed that $\alpha(n)$ is essentially a constant, like $\alpha(n) \leq 4$ for all "practical" values of $n$, see Pach and Sharir [26].

The problem of estimating $\lambda_{s}(n)$ for $s>4$ is more complicated. For any constant $s$, it is well-known the bound $\lambda_{s}(n)=O\left(n \log ^{*} n\right)$. Recall that $\log ^{*} n$ is the minimum number of times $q$ such that $q$ consecutive applications of the $\log$ operator will map $n$ to a value smaller than 1 , i.e., (q)

$\overbrace{\log \ldots \log } n \leq 1$. Actually, $\log ^{*} n$ is the smallest height of an exponential "tower" of 2 's, $2^{2^{2 \cdots}}$ which is $\geq n$ (nothing changes if 2 is replaced by another base $b>1$ ). Observe that, $\log ^{*} n$ is much smaller than $\log n$ and it can be considered 
almost constant for "practical" values of $n$, see Sharir and Agarwal [29], Pach and Sharir [26].

\section{Acknowledgments}

The authors thank Prof. J. A. Mesa for his interesting comments on preliminary versions of the article. They also thank three anonymous referees for very constructive reports that have led to improve the results of the article.

\section{REFERENCES}

[1] I. Averbakh, On the complexity of a class of combinatorial optimization problems with uncertainty, Math Program 90 (2001), 263-272.

[2] I. Averbakh, Complexity of robust single facility location problems on networks with uncertain edge lengths, Discr Appl Math 127 (2003), 505-522.

[3] I. Averbakh, The minmax relative regret median problem on networks, INFORMS J Comput 17 (2005), 451-461.

[4] I. Averbakh and O. Berman, Minmax regret p-center location on a network with demand uncertainty, Location Sci 5 (1997), 247-254.

[5] I. Averbakh and O. Berman, Minmax regret median location on a network under uncertainty, INFORMS J Comput 12 (2000), 104-110.

[6] I. Averbakh and O. Berman, Algorithms for the robust 1center problem on a tree, Eur J Oper Res 123 (2000), 292-302.

[7] I. Averbakh and O. Berman, An improved algorithm for the minmax regret median problem on a tree, Networks 41 (2003), 97-103.

[8] I. Averbakh and V. Lebedev, Interval data minmax regret network optimization problems, Discr Appl Math 138 (2004), 289-301.

[9] O. Berman and E.H. Kaplan, Equity maximizing facility location schemes, Transp Sci 24 (1990), 137-144.

[10] R.E. Burkard and H. Dollani, Robust location problems with Pos/Neg weights on a tree, Networks 38 (2001), 102113.

[11] R.E. Burkard and H. Dollani, A note on the robust 1-center problem on trees, Ann Oper Res 110 (2002), 69-82.

[12] E. Erkut, Inequality measures for location problems, Location Sci 1 (1993), 199-217.

[13] M.T. Caceres, M.C. Lopez-de-los-Mozos, J.A. Mesa, J. Puerto, and A.M. Rodriguez-Chia, "Robust mean absolute deviation problems on tree networks," Proc XVIII EWGLA Meeting, Naples, Italy, 2010, pp. 89.
[14] B. Chen and C.S. Lin, Minmax regret robust 1-median location on a tree, Networks 31 (1998), 93-103.

[15] R.L. Church and R.S. Garfinkel, Locating an obnoxious facility on a network, Transport Sci 12 (1978), 107-118.

[16] E. Conde, Minmax regret location-allocation problem on a network under uncertainty, Eur J Oper Res 179 (2007), 1025-1039.

[17] E. Conde, A note on the minmax regret centdian location on trees, Oper Res Lett 36 (2008), 271-275.

[18] H. Edelsbrunner, L. Guibas, J. Pach, R. Pollack, R. Seidel, and M. Sharir, Arrangements of curves in the plane-topology, combinatorics, and algorithms, Theor Comput Sci 92 (1992), 319-336.

[19] E. Erkut and B.C. Tansel, On parametric medians of trees, Transp Sci 26 (1992), 149-156.

[20] P. Kouvelis and G. Yu, Robust discrete optimization and its applications, Kluwer, Boston, 1997.

[21] M. McKenna and J. O'Rourke, "Arrangements of lines in 3-space: A data structure with applications," Proc Ann 4th ACM Symp Comput Geometry, Urbana-Champaign, IL, USA, pp. 371-380.

[22] M.T. Marsh and D.A. Schilling, Equity measurement in facility location analysis: A review and framework, Eur J Oper Res 74 (1994), 1-17.

[23] J.A. Mesa, J. Puerto, and A. Tamir, Improved algorithms for several location problems with equality measures, Discr Appl Math 130 (2003), 437-448.

[24] W. Ogryczak, Inequality measures and equitable locations, Ann Oper Res 1 (2009), 61-86.

[25] W. Ogryczak, Conditional median as a robust solution concept for uncapacitated location problems, TOP 18 (2010), 271-285.

[26] J. Pach and M. Sharir, Combinatorial geometry and its algorithmic applications, The Alcalá Lectures, AMS: Math Surv Monographs 152 (2009).

[27] J. Puerto, F. Ricca, and A. Scozzari, Minimax regret path location on trees, Accept Networks 58 (2011), 147-158.

[28] J. Puerto, A.M. Rodriguez-Chia, and A. Tamir, Minimax regret Single facility ordered median location problems on networks, INFORMS J Comput 21 (2009), 77-87.

[29] M. Sharir and P.K. Agarwal, Davenport-Schinzel sequences and their geometric applications, Cambridge University Press, New York, NY, 1995.

[30] A. Tamir, On the complexity of some class of location problems, Transp Sci 26 (1992), 352-354.

[31] G.L. Vairaktarakis and P. Kouvelis, Incorporation dynamic aspects and uncertainty in 1-median location problems, Nav Res Logist 46 (1999), 147-168. 